\title{
Effect Analysis of PDT and VAD Regimen in the Treatment of Multiple Myeloma
}

\author{
Jun Sun, Bo Ma, Xinyu Cui, Jie Bao, Lei Lei, Ling Li, Lili Bi, Lina Wang
}

The Third Affiliated Hospital of Qiqihar Medical University, Qiqihar, Heilongjiang, 161000

Keywords: Multiple Myeloma; PDT; VAD

\begin{abstract}
Objective: To compare the clinical efficacy of pirarubicin, dexamethasone plus thalidomide (PDT regimen) with vincristine, doxorubicin plus dexamethasone (VAD regimen) in patients with multiple myeloma (MM). Methods: Thirty-six patients with multiple myeloma were randomly divided into the control group $(n=18)$ and the observation group $(n=18)$. The control group was treated with PDT. The observation group was treated with VAD. After treatment, the therapeutic effects of the two groups were compared. Result: The total effective rate is $66.67 \%$ in the PDT group and $61.11 \%$ in the VAD group. There was no statistical significance $(\mathrm{P}>0.05)$. The adverse reactions in the PDT group were smaller than those in the VAD group, and the difference was statistically significant $(\mathrm{P}<0.05)$. Conclusion: The PDT regimen is a treatment for multiple myeloma. The therapeutic effect is better, and the incidence of cardiotoxicity and peripheral neuritis is significantly reduced. Further improvement of various clinical indicators is worthy of clinical promotion.
\end{abstract}

\section{Introduction}

Multiple myeloma (MM) is a malignant clonal proliferative disease of plasma cells, which occurs mostly in middle-aged and elderly people. Its incidence rate accounts for $1 \%$ and $10 \%$ of all malignant tumors and hematological tumors, respectively, and is increasing year by year. trend. At present, combined chemotherapy is the main means of treatment of MM. Although the chemotherapy regimen has been continuously improved and the intensity of chemotherapy has been continuously improved, it has not achieved satisfactory results. Our department mainly used VAD chemotherapy before 2012. This program has a large peripheral neuritis and cardiotoxicity, and then adjusted to PDT chemotherapy. Now we compare the efficacy and side effects of the two regimens.

\section{Materials and Methods}

\subsection{Clinical Data.}

Eighteen patients were treated with PDT regimen, and the median course of treatment was 6 (3-10). All patients met the diagnostic criteria for multiple myeloma in the Blood Disease Diagnosis and Efficacy Criteria [1]. There were 12 males and 6 females with an average age of 63 (54-76) years. Eighteen patients were treated with the VAD regimen. The median duration of treatment was 5 (2-9), including 10 males and 8 females with an average age of 61 (51-75) years. All patients were diagnosed as MM by routine blood, biochemistry, radiation, pathology or cytology. Clinical staging was performed according to Durie and Salmon criteria.

\subsection{Methods.}

PDT group: pirarubicin $10 \sim 15 \mathrm{mg}$, 1st to 4th day; dexamethasone $20 \sim 40 \mathrm{mg}$, 1st to 4th, 9th to 12th, 17th to 20th day; thalidomide (Thalidomide) $5 \sim 100 \mathrm{mg} / \mathrm{d}, 1 \sim 28$ days; $28 \mathrm{~d}$ is a course of treatment. VAD group: vincristine (VCR) $0.4 \mathrm{mg}$, days 1 to 4 ; doxorubicin (ADM) $10 \mathrm{mg}$, days 1 to 4; DXMS 40 mg, days 1 to 4, days 9 to 12, 17 to 20 days; 28 days is a course of treatment. During chemotherapy, symptomatic supportive treatment is given according to the patient's condition, and if necessary, G-CSF, blood product infusion, etc. Blood, liver and kidney function, myocardial 
enzyme, electrocardiogram, immunoglobulin, blood calcium, bone X-ray examination, serum immunofixation electrophoresis, etc. were observed regularly before and after treatment.

\subsection{Efficacy Evaluation Criteria.}

After 2 to 3 courses of chemotherapy, the efficacy was evaluated based on clinical manifestations and various indicators. The efficacy criteria were carried out according to the literature [1]. Remission includes complete remission (CR) and partial remission (PR); non-remission (NR) includes stable and progression. Effectiveness $=(\mathrm{CR}$ number of cases + number of PR cases $) /$ total number of cases $\times 100 \%$.

\subsection{Statistical Analysis.}

The data of this study were all processed by SPSS20.0 statistical software. The count data was expressed as a percentage. The comparison between groups was performed by $\chi 2$ test, $\mathrm{P}<0.05$ was considered statistically significant.

\section{Results}

\subsection{Comparison of Curative Effect.}

In the PDT group, the effective rate was $66.9 \%$ and VAD was $65.4 \%$ after three courses of treatment. There was no significant difference between the two groups $(\mathrm{P}>0.05)$, as shown in Table 1.

Table 1 Comparison of the efficacy of PDT and VAD in the treatment of MM

\begin{tabular}{clllll} 
Group rate & \multicolumn{2}{l}{ Number of } & PR & NR & Efficient [n(\%)] \\
\hline PDT & 18 & 1 & 11 & 6 & $12(66.67)$ \\
VAD & 18 & 1 & 10 & 7 & $11(61.11)$ \\
\hline
\end{tabular}

\subsection{Comparison of Adverse Reactions.}

The adverse reactions of cardiotoxicity, alopecia and peripheral neuritis in the PDT group were significantly less than those in the VAD group. The difference was statistically significant $(\mathrm{P}<0.05)$, see Table 2.

Table 2 PDT and VAD treatment MM adverse reactions comparison [n (\%)]

\begin{tabular}{lllc}
\hline Comparison value & PDT group(n=18) & VAD group(n=18) & $\mathrm{P}$ \\
\hline Elevated myocardial enzyme & $3(16.7)$ & $9(50.0)$ & $>0.05$ \\
WBC reduction (level 3-4) & $2(11.1)$ & $3(16.7)$ & $>0.05$ \\
Plt reduction (3-4 levels) & $2(11.1)$ & $3(16.7)$ & $<0.05$ \\
ECG change & $4(22.2)$ & $9(50.0)$ & $<0.05$ \\
Liver function damage & $3(16.7)$ & $5(27.8)$ & $<0.05$ \\
Peripheral neuritis & $1(5.6)$ & $8(44.4)$ & $>0.05$ \\
Gastrointestinal reaction & $7(33.3)$ & $7(38.9)$ & $>0.05$ \\
Hair loss & $6(33.3)$ & $12(66.7)$ & $<0.05$ \\
\hline
\end{tabular}

\section{Discussion}

Multiple bone marrow is a malignant clonal disease of plasma cells commonly found in elderly patients. With the aging of the population structure in China, the incidence rate has increased. In recent years, due to the development of hematopoietic stem cell transplantation technology and the 
use of bortezomib and lenalidomide, it has brought good therapeutic effects to patients with multiple myeloma. However, due to the age of the patient, poor physical condition and economic factors, the use of the above methods and drugs is largely restricted. MM is still an incurable disease, the main treatment is combined chemotherapy, commonly used programs are MP, VAD, M2, of which VAD program is a better solution for the treatment of MM. The VAD regimen is a more traditional approach to the treatment of multiple myeloma, but cardiotoxicity and peripheral neuritis limit its widespread use. Pirarubicin is a semi-synthetic anthracycline antineoplastic drug synthesized in 1979 and derived from doxorubicin. Compared with doxorubicin, pirarubicin has less cardiotoxicity and adverse reactions to hair loss. Thalidomide is a glutamic acid derivative, which is used in the treatment of multiple myeloma by anti-angiogenesis, immune mediation, induction of apoptosis [2], etc., which can overcome the resistance of previous chemotherapy drugs. It has no bone marrow suppression toxicity and has a good therapeutic effect on newly diagnosed, refractory and relapsed multiple myeloma.

This article compares the efficacy and adverse effects of PDT and VAD regimens in the treatment of patients with multiple myeloma. The results showed that there was no significant difference in the efficacy of the two regimens, but the adverse events in the PDT group were small. This conclusion is consistent with the literature [3-4], indicating that the replacement of doxorubicin with pirarubicin can significantly reduce the adverse effects of the drug on the heart. The use of vincristine in the PDT regimen significantly reduced the incidence of peripheral neuritis and had a significant effect on improving patient quality of life and improving treatment compliance. This is also consistent with the "reduction of vincristine" in the recommended regimen of the latest guidelines [5-6]. In summary, the PDT regimen can treat multiple myeloma, and alleviate adverse reactions on the basis of consistent treatment effects.

\section{References}

[1] Zhang Zhinan, Shen Wei. Diagnosis and efficacy criteria of blood diseases [M]. Beijing: Science Press, 2007: 87-89. [2] Zhang Yugao, Han Liying. Anti-tumor mechanism and clinical application of reaction stop [J]. Medical Review, 2007, 13 (3): 200-202.

[3] Guo Chao, Li Zhichun. Clinical comparison of pirarubicin and doxorubicin in the treatment of multiple myeloma [J].Journal of Clinical Medicine, 2005, 25(8):27-29.

[4] Hou Jian, Fu Weijun, Yuan Zhengang, et al. Comparative study of pirarubicin and doxorubicin in the treatment of multiple myeloma[J].Journal of Leukemia \& Lymphoma,2008,17(3):209-210 .

[5] Chen Wenming. Guidelines for the diagnosis and treatment of multiple myeloma in China (revised in 2013) [J]. Chinese Journal of Internal Medicine, 2013, 52 (009): 797-798.

[6] Su Nan, Yu Jinxiang, Li Yan, et al. Clinical observation of bortezomib combined with dexamethasone in the treatment of stage III multiple myeloma [J]. Chinese Journal of Clinical Oncology and Rehabilitation, 2009, 16(2): 146-150. 